\title{
RESEARCH
}

Open Access

\section{The dynamics of biomarkers across the clinical spectrum of Alzheimer's disease}

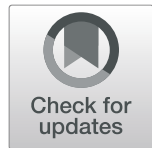

\author{
Christoforos Hadjichrysanthou ${ }^{1 *} \mathbb{D}$, Stephanie Evans ${ }^{1,2}$, Sumali Bajaj ${ }^{1}$, Loizos C. Siakallis ${ }^{3}$, Kevin McRae-McKee', \\ Frank de Wolf ${ }^{1}$, Roy M. Anderson ${ }^{1}$ and the Alzheimer's Disease Neuroimaging Initiative
}

\begin{abstract}
Background: Quantifying changes in the levels of biological and cognitive markers prior to the clinical presentation of Alzheimer's disease (AD) will provide a template for understanding the underlying aetiology of the clinical syndrome and, concomitantly, for improving early diagnosis, clinical trial recruitment and treatment assessment. This study aims to characterise continuous changes of such markers and determine their rate of change and temporal order throughout the AD continuum.

Methods: The methodology is founded on the development of stochastic models to estimate the expected time to reach different clinical disease states, for different risk groups, and synchronise short-term individual biomarker data onto a disease progression timeline. Twenty-seven markers are considered, including a range of cognitive scores, cerebrospinal (CSF) and plasma fluid proteins, and brain structural and molecular imaging measures. Data from 2014 participants in the Alzheimer's Disease Neuroimaging Initiative database is utilised.

Results: The model suggests that detectable memory dysfunction could occur up to three decades prior to the onset of dementia due to AD (ADem). This is closely followed by changes in amyloid- $\beta$ CSF levels and the first cognitive decline, as assessed by sensitive measures. Hippocampal atrophy could be observed as early as the initial amyloid- $\beta$ accumulation. Brain hypometabolism starts later, about 14 years before onset, along with changes in the levels of total and phosphorylated tau proteins. Loss of functional abilities occurs rapidly around ADem onset. Neurofilament light is the only protein with notable early changes in plasma levels. The rate of change varies, with CSF, memory, amyloid PET and brain structural measures exhibiting the highest rate before the onset of ADem, followed by a decline. The probability of progressing to a more severe clinical state increases almost exponentially with age. In accordance with previous studies, the presence of apolipoprotein E4 alleles and amyloid- $\beta$ accumulation can be associated with an increased risk of developing the disease, but their influence depends on age and clinical state.
\end{abstract}

Conclusions: Despite the limited longitudinal data at the individual level and the high variability observed in such data, the study elucidates the link between the long asynchronous pathophysiological processes and the preclinical and clinical stages of AD.

Keywords: Alzheimer's disease, Dementia, Biomarker trajectories, Clinical states, Time to event, Markov chain, ADNI, CSF, PET, Plasma, Brain

\footnotetext{
* Correspondence: c.hadjichrysanthou@imperial.ac.uk

'Department of Infectious Disease Epidemiology, School of Public Health, Imperial College London, London, UK

Full list of author information is available at the end of the article
}

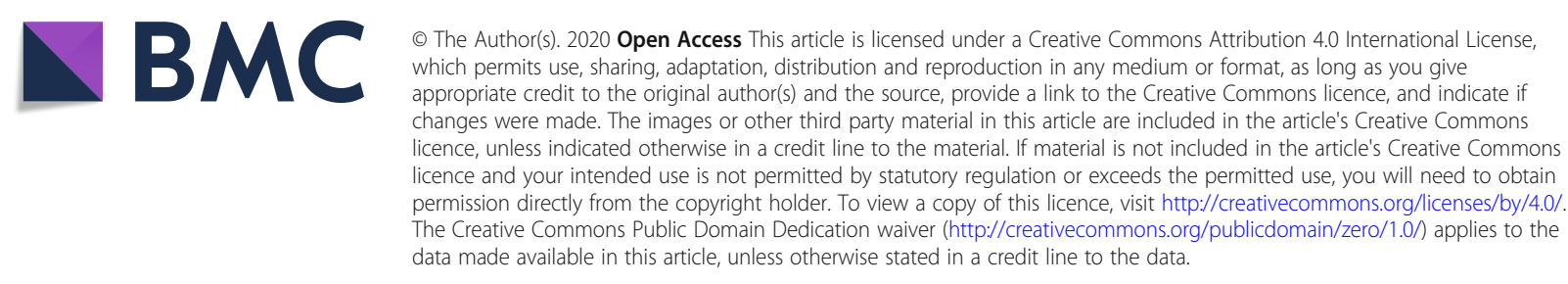




\section{Background}

Alzheimer's disease (AD) is a neurodegenerative disorder with long preclinical and prodromal stages $[1,2]$, where pathophysiological changes, and potentially irreversible brain damage, occur many years prior to clinical manifestation. Despite the problems and practicalities of collecting frequent biomarker data over a long period of time, research over the last two decades has improved our understanding of the natural history of $\mathrm{AD}$ and demonstrated the importance of considering the disease as a multifaceted process moving along a biological and clinical continuum [3].

To date, there is limited longitudinal data for individuals with repeated biomarker measures throughout the AD continuum. Hence, descriptions of the continuous, temporal dynamics of potential biological and clinical markers during the progression of individuals from healthy stages to dementia due to $\mathrm{AD}$ (ADem) are mainly based on highly heterogeneous data collected over a relatively short period of time. Previous work has mainly focused on the description of the dynamics within different clinical states $[4,5]$. In studies of autosomal dominant $\mathrm{AD}$, early pathologic changes can be described as a function of the expected time to symptoms onset. This time is predicted based on the age of the individual and a retrospective estimate of the age at which the parent developed $\mathrm{AD}$ symptoms [6]. Estimates in these studies have also been produced based on the mean age at onset of all other individuals with the same autosomal dominant $\mathrm{AD}$ mutation type [7]. In the common form of sporadic $\mathrm{AD}$, estimating the time required to reach a disease state is more challenging [8]. Techniques that have been employed for the prediction of the dynamics of biomarkers include the synchronisation of individual data based on a disease progression score, which can be a function of the individual's age $[9,10]$, and the optimal alignment of individuals' biomarker trajectories with the estimated long-term population-level trajectory $[11,12]$. Time-dependent changes of potential biomarkers have also been characterised on different scales of disease progression based on the individual biomarker rate of change as estimated from the short-term longitudinal observations [2, 13-15]. Other data-driven progression models have recently emerged for the description of disease progression without the need of a priori staging of individuals [13].

Although the description of the evolution of potential biomarkers without relying on pre-defined clinical stages has its own merits, the diagnosis of AD based exclusively on the presence of abnormal levels of specific biomarkers is still under research [16]. On the other hand, categorising individuals using clinical syndromic criteria is useful in clinical practice [17]. Despite the challenges and limitations, this categorisation has been helpful in clinical and epidemiological studies, including the evaluation and standardisation of individuals' clinical state and the assessment of the public health impact and cost of care.

In this study, we aim to describe the pathophysiological changes that may be observed during the clinical progression from cognitively healthy stages to Alzheimer's clinical syndrome [16] and provide further insights into their temporal relation. Based on the clinical diagnosis and a set of demographic and genetic factors, we develop a stochastic model to estimate the expected time required for an individual at a specific clinical state to reach any of the more severe states. Three commonly accepted clinical states are considered: the cognitively normal state $(\mathrm{CN})$, the mild cognitive impairment state (MCI) and the ADem state [16, 18]. Two established major risk factors for sporadic Alzheimer's are taken into account: the presence of the apolipoprotein $\mathrm{E} \varepsilon 4$ genotype (APOE $\varepsilon 4$ ) [19] and age. We also extend the model to consider the effect of educational attainment [20]. Individual data from a range of cognitive and pathologically relevant disease markers are aligned based on their temporal distance from a disease state. Assuming that similar biological and cognitive changes occur in all individuals within a defined risk group, the population-level dynamics of markers and a temporal order of changes are estimated. The focus is on the link between dynamic pathophysiological changes and the clinical presentation of various clinical stages of $\mathrm{AD}$.

\section{Methods \\ Clinical progression \\ The model}

We developed a Markov model to estimate the expected time needed for different risk groups to reach a given clinical state, provided individuals will eventually develop ADem, i.e. that death is not a competing event and ADem is the only absorbing state.

Let ${ }^{a} I^{\{S\}}$ denote the group of individuals at age $a$ that have a set of time-invariant characteristics $\{S\}$. Individuals ${ }^{a} I^{\{S\}}$ that have been clinically classified in state A can transition to state B within the next year with probability $a p_{A, B}^{\{S\}}$. The possible transitions between the three clinical states, $\mathrm{CN}, \mathrm{MCI}$ and $\mathrm{ADem}$, are as shown in the schematic diagram in Fig. 1 [21, 22].

\section{Transition probabilities}

Generalised linear mixed models (GLMMs) with a logit link function were developed and fitted, and then the probability of transitioning from state A to state B was estimated conditional on the covariates [23]. The main model incorporated four covariates, namely, age (agesquared) at the point of transition to a clinical state 


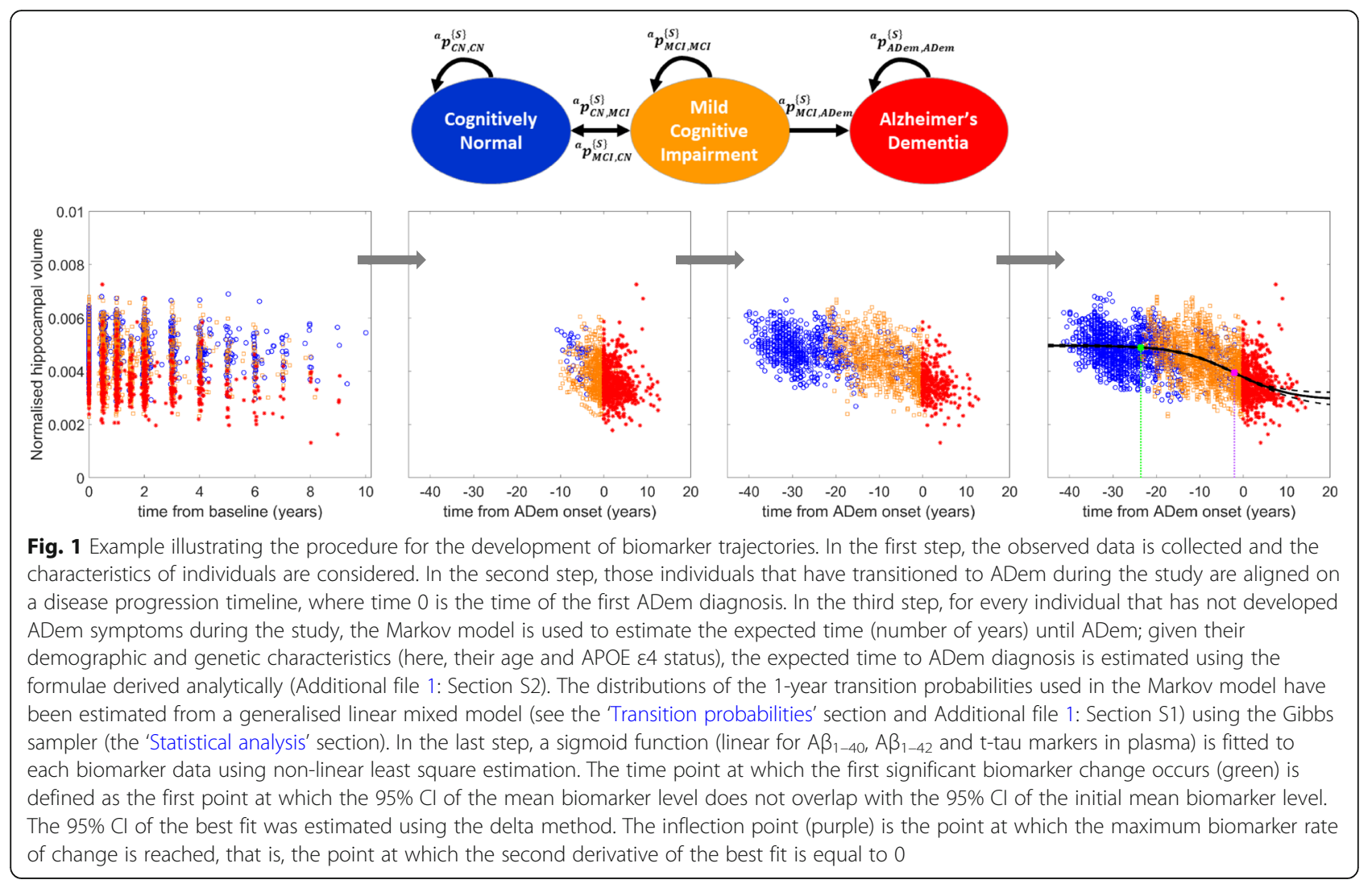

(continuous, centred at 50 years and scaled by a factor of 100 to normalise), APOE $\varepsilon 4$ status (binary, presence/absence of APOE $\varepsilon 4$ alleles), time between clinic visits in years (centred at 12 months) and the current diagnostic state (CN, MCI, ADem). An interaction term between age-squared and diagnostic state was also included. We also explored the significance of an interaction term between APOE $\varepsilon 4$ status and age in the model. While several studies have reported that APOE \&4-related risk for developing ADem may vary significantly with age [24, $25]$, in the sample dataset we considered it has been shown that this term is not significant $(p>0.7$, checked at the $5 \%$ level of significance) and the model without the interaction term provides better fits to the data. When the educational level was incorporated into the model, this was defined dichotomously based on the number of years of education; $\leq 16$ or $>16$. The choice of 16 years as the cut-off point in education has been made based on the median (=16) and mean $(=16.04)$ of the years of education in the sample. Details of the GLMM are provided in Additional file 1: Section S1.

\section{Expected time to $\mathrm{MCl}$ and ADem}

Given the demographic and genetic characteristics of individuals at the time point of each measurement, and the respective transition probabilities from that point, the expected time required to reach a more severe state was estimated using the formulae derived from the stochastic model, presented in Additional file 1: Section S2.

\section{Marker trajectories \\ Development}

We assumed that during the development and progression of the disease, all individuals in a defined risk group (see the 'Dataset: the Alzheimer's Disease Neuroimaging Initiative' section) present similar cognitive and biological dynamic changes. First, the measurements from individuals that have developed ADem during the study were aligned on a disease progression timeline, where time 0 is the time of ADem onset (visit at which ADem was first diagnosed). In order to provide insight into the behaviour of the markers after ADem onset, measurements of individuals that were diagnosed with ADem at their first visit have also been included by using the best estimate of the year of onset of ADem symptoms as reported in the data (years from ADem onset $\approx$ exam date - estimated date of onset, available in ADNI-1 and ADNI-GO). Following the calculation of the expected time to ADem from each measurement of $\mathrm{CN}$ and $\mathrm{MCI}$ individuals that have not developed ADem during the study, all their biomarker data were also aligned on the same disease timeline (see Fig. 1). Based on conceptual models and current evidence in the literature $[9,26]$, it was assumed that markers are monotonically decreasing 
or increasing during the disease progression following a non-linear temporal course, and in particular, a sigmoidal type pattern. This excludes the $A \beta_{1-40}, A \beta_{1-42}$ and total tau ( $\mathrm{t}$-tau) markers in plasma which, due to small changes during the clinical progression, were described by a linear model of the form $B\left(t_{\text {ADem }}\right)=r_{1}+$ $r_{2} t_{\mathrm{ADem}}$, where $t_{\mathrm{ADem}}$ (independent variable) is the time from $\mathrm{ADem}$, and $t_{\mathrm{ADem}}=0$ corresponds to the time of the first ADem diagnosis. A sigmoid function of the following form was fitted to each dataset for each other marker $B$ :

$$
B\left(t_{\mathrm{ADem}}\right)=r_{1}+\frac{r_{2}-r_{1}}{1+\exp \left(r_{3}\left(t_{\mathrm{ADem}}-r_{4}\right)\right)} .
$$

We restricted $r_{3}<0$, so $r_{1}$ is the value that $B\left(t_{\mathrm{ADem}}\right)$ approaches to as $t_{\mathrm{ADem}} \rightarrow-\infty$, and $r_{2}$ is the value that $B\left(t_{\mathrm{ADem}}\right)$ approaches to as $t_{\mathrm{ADem}} \rightarrow \infty$.

\section{Normalisation}

Changes in the potential AD markers have been considered collectively on the same scale by using the minmax normalisation method, so that the normalised form, $B_{\text {norm }}$, of the mean trajectory $B$ is given by:

$$
B_{\text {norm }}\left(t_{\mathrm{ADem}}\right)=\left|\frac{B\left(t_{\mathrm{ADem}}\right)-\lim _{t_{\mathrm{ADem}} \rightarrow-\infty} B\left(t_{\mathrm{ADem}}\right)}{\lim _{t_{\mathrm{ADem}} \rightarrow-\infty} B\left(t_{\mathrm{ADem}}\right)-\lim _{t_{\mathrm{ADem}} \rightarrow+\infty} B\left(t_{\mathrm{ADem}}\right)}\right| .
$$

$\lim _{t_{\mathrm{ADem}} \rightarrow-\infty} B_{\text {norm }}\left(t_{\mathrm{ADem}}\right)=0$ is thus the healthiest level (normal), and $\lim _{t_{\mathrm{ADem}} \rightarrow+\infty} B_{\text {norm }}\left(t_{\mathrm{ADem}}\right)=1$ is the most severe level (abnormal). The limits of $B\left(t_{\mathrm{ADem}}\right), \lim _{t_{\mathrm{ADem}} \rightarrow-\infty} B($ $\left.t_{\mathrm{ADem}}\right)$ and $\lim _{t_{\mathrm{ADem}} \rightarrow+\infty} B\left(t_{\mathrm{ADem}}\right)$ are fixed to the respective limits of the best fit of $B\left(t_{\mathrm{ADem}}\right)$.

\section{First biomarker change, rate of change and inflection point}

We considered the time of the first significant biomarker change to be the first time point at which the $95 \%$ confidence interval (CI) of the predicted mean biomarker level does not overlap with the $95 \%$ CI of the predicted mean value before any change (the lowest or the highest mean value, depending on whether the biomarker monotonically increases or decreases). For each normalised marker trajectory, the instantaneous rate of change, which represents the change in the marker level at each instant in time, and the inflection point, which is the point at which the maximum rate is observed before start declining, have also been considered. These were derived by calculating the first and the second derivative, respectively, of the equation describing the trajectory.

\section{Markers considered}

We focused our study on a number of markers that, alone or with other markers, could serve as indicators of $\mathrm{AD}$ progression and/or endpoints in clinical trials. Twenty-seven markers have been considered in total. The markers can be classified into four main categories: markers of cognitive performance, biological markers that can be measured in cerebrospinal (CSF) and plasma fluid, and neuroimaging markers.

\section{Cognitive markers}

Two AD Assessment Scale-Cognitive Subscale tests which serve as $\mathrm{AD}$ and dementia indicators: the ADASCog 11 (ranged from 0 to 70 , with the first number being the least severe and the last the most severe [27]) and the ADAS-Cog 13 (0 to 85) [28]; the Mini-Mental State Examination (MMSE) test (30 to 0) [29] which is an indicator of mental status; the Clinical Dementia Rating Scale-Sum of Boxes (CDR-SB) (0 to 18) [30, 31]; the Montreal Cognitive Assessment (MoCA) (30 to 0) [32]; and the Functional Activities Questionnaire (FAQ), concerned with performing daily tasks necessary for independent living ( 0 to 30 , with higher scores reflecting greater dependency) [33]. Two efficient neuropsychological instruments for evaluating different aspects of episodic memory have also been considered: the Rey's Auditory Verbal Learning Test (RAVLT) [34] (here, we focused on RAVLT-Immediate recall scores (RAVLT Immediate, 75 to 0$)$ ), and the Logical Memory-Delayed recall scores (LM_Delayed, 25 to 0) [35]. Additionally, we investigated the change in two composite scores, each of which is a weighted combination of components of well-validated neuropsychological tests. The first is the Preclinical Alzheimer Cognitive Composite [36] (PACC, the modified version of [37] to include the Trail Making Test B), which has been designed to serve as the primary outcome measure for trials conducted at the asymptomatic phase of the disease (lower scores indicate more severe impairment). The second is the AD Composite Score (ADCOMS) (0 to 1.97) [38], which has been developed to detect a related clinical decline in amnestic $\mathrm{MCI}$ due to $\mathrm{AD}$, and prodromal and mild $\mathrm{AD}$.

\section{CSF markers}

The 42-amino acid form of amyloid $\beta$ protein, $A \beta_{1-42}$, which indicates brain amyloid pathology [39]; the neurofilament light (NfL), a biomarker of neuroaxonal injury or neurodegeneration [40]; the total tau ( $\mathrm{t}$-tau) and phosphorylated tau (p-tau) proteins [39], which can be considered as potential biomarkers of Alzheimer-type axonal degeneration and neurofibrillary tangle formation, respectively; and neurogranin $(\mathrm{Ng})$, which has also recently emerged as a potential synaptic biomarker [41]. 


\section{Plasma fluid markers}

$\mathrm{A} \beta_{1-40} ; \mathrm{A} \beta_{1-42} ; \mathrm{NfL} ; \mathrm{t}$-tau.

\section{Neuroimaging markers}

The MRI volumetric measurements of the following brain regions have been considered: hippocampus, whole brain, entorhinal cortex, fusiform gyrus and middle temporal gyrus [42]. To account for the inter-individual variation in head size, all volumes of interest have been normalised for total intracranial volume (ICV) [43], producing a unitless value between 0 and 1 . We also considered ${ }^{18} \mathrm{~F}$-fluorodeoxyglucose (FDG) positron emission tomography (PET) which is used to visualise changes in the cerebral glucose metabolism [44], and standardised uptake value ratio (SUVr) for Pittsburgh compound B (PiB)-PET [45] and florbetapir (F18-AV-45) PET [46], which both allow in vivo assessment of cerebral amyloid load.

\section{Dataset: the Alzheimer's Disease Neuroimaging Initiative} The dataset used was obtained from the Alzheimer's Disease Neuroimaging Initiative (ADNI) database (adni. loni.usc.edu, last downloaded on September 1, 2018). ADNI is a multisite (63 ADNI clinical trial sites are located across the USA and Canada) longitudinal study of normal cognitive ageing, $\mathrm{MCI}$ and early $\mathrm{AD}$ individuals. It has four phases: ADNI-1, ADNI-GO, ADNI-2 and ADNI-3. ADNI is not a population-based study; its population represents a primarily amnestic clinical population that can be used in treatment trials. Participants were recruited following specific recruitment methods. The procedure of ADem clinical diagnosis follows the standardised clinical criteria as outlined by the NINCDS-ADRDA guidelines [18]. For a detailed list of all diagnostic, and inclusion and exclusion criteria, readers are referred to the Procedures Manual (http:// adni.loni.usc.edu/methods/documents).

The original dataset obtained from ADNI consists of 2066 individuals. The study duration up to the time the current analysis was performed is about 12 years. Repeated assessments took place every 6 months for the first 2 years and yearly afterwards. Two hundred fiftyfive participants (from ADNI-2) were categorised as 'significant memory concern' (SMC) at baseline, and in the current analysis, were combined with individuals diagnosed as $\mathrm{CN}$; the key inclusion criteria that distinguish SMC are a self-report significant memory concern from the participant, although participants in this class scored within the normal range for cognition (or $\mathrm{CDR}=0$ ). However, this is not equated as progressive memory impairment or as consistent forgetfulness. In addition, in ADNI-2, MCI is divided further into 'early MCI' (EMCI) and 'late MCI' (LMCI), and in this analysis, the MCI subtypes were combined. Thirty-two individuals that had only a screening visit/diagnosis (preceding the baseline visit/diagnosis) were excluded from the analysis. Out of the 698 individuals that developed dementia, 20 developed non-Alzheimer's dementias and were also removed.

For the prediction of clinical progression and calculation of the transition probabilities, we used only those individuals that had at least two visits with clinical diagnosis. For the development of a marker trajectory in a specific risk group (e.g. those that reached abnormal amyloid levels), all available individual-level measurements in that group were utilised, given that all information for the calculation of the expected time to ADem from the point of measurement was available. The trajectory of each marker was first estimated independently for the entire sample (Additional file 1: Section S3, Fig. S1); the ADNI dataset consists of a highly selective clinical population that can be considered as a good representation of a population that will most likely be affected by AD. The same method was then applied for only those individuals that have shown some evidence of substantial $A \beta$ in their brain (amyloid-positive individuals), and so have an increased chance of being in the Alzheimer's continuum [12, 16]. In particular, individuals that have ever crossed one of the following thresholds have been considered as amyloid-positive individuals: CSF $\mathrm{A} \beta_{1-42}=880 \mathrm{pg} / \mathrm{mL}$ [47] or $/$ and global PET florbetapir $\mathrm{SUVr}=1.11$, normalised to the whole cerebellum [48], or/and PiB-PET SUVr (standardised in the region relative to cerebellar grey matter) $=1.5[2,49,50]$. For the development of the trajectories in the subset of amyloid-positive individuals, the transition probabilities were re-calculated in this group.

To make a comparison of the times at which the first significant changes and the inflection points of biomarkers occur, the analysis was performed on a reduced dataset. The complete data for all biomarkers was not sufficient to support the model and provide good estimations for all biomarkers. As a compromise, we have chosen only the measurements at the visits where CSF $A \beta_{1-42}$ was available; CSF $A \beta_{1-42}$ is one of the markers with the lowest number of measurements, and many of the other markers have also been measured when CSF $\mathrm{A} \beta_{1-42}$ was measured (see Additional file 1: Section S3, Table S1). Data of CSF NfL and Ng, the plasma markers and PiB-PET could not always support the sigmoid function and have been excluded from the comparison with other markers of disease progression.

\section{Statistical analysis}

The transition probabilities of the model were estimated using the Gibbs sampler, which is a Markov chain 
Monte Carlo (MCMC) algorithm. It was assumed that all parameters had improper uniform prior distributions, and convergence of the MCMC algorithms was assessed visually from the traces of each parameter. A number of GLMMs consisting of different combinations of variables have been considered. The model selection procedure was based on the deviance information criterion (DIC). The MCMC algorithms and goodness of fit statistics were implemented within the runMLwiN function from the R2MLwiN package in R [51].

The models of biomarker dynamics were fitted using iterative non-linear least square estimation, by initiating each step using the best parameter estimates of the previous step until the parameter estimates are optimised. When fitting the model, we took into account any known lower and upper limits of the markers, and we restricted $r_{1}, r_{2}$ and the prediction intervals accordingly so that the estimated values fall within these limits. For markers for which these limits are unknown, the lowest and highest values observed in the original dataset were used. The biomarker trajectories and all figures in the study were produced using MATLAB R2019a.

The $95 \% \mathrm{CI}$ of all quantities have been computed using the 2.5th and 97.5th percentiles of the distribution obtained by performing the analysis on 500 bootstrap samples (produced by random sampling with replacement from the observations in the original sample used in each case, so that the number of measurements at each clinical state was equal to that of the original sample). For each bootstrap sample, probabilities of transitioning to different states were drawn at random from the respective probability distributions produced using the MCMC algorithm. The expected times to ADem onset were calculated for every individual in the sample, and the model of biomarker dynamics was fitted to it. The delta method was used to estimate the $95 \%$ CI of the best fit in each iteration of bootstrap resampling, which in turn was used for the calculation of the first significant biomarker change. For each marker, and each time point, we also estimated the $95 \%$ prediction interval for new observations (non-simultaneous bounds), which indicates the confidence that the new observation will lie within that interval given a single observation time point (predictor value). In addition to quantifying model uncertainty, further analysis has been performed to evaluate the performance of the model, including a single train-and-test experiment (holdout method), where the dataset of individuals that were $\mathrm{CN}$ at baseline and developed $\mathrm{MCI}$ and ADem during the study serves as the test set (Additional file 1: Section S7).

\section{Results}

Individuals were removed from the dataset subject to the constraints described in the 'Methods' section, which yielded data from 2014 individuals. The baseline composition and characteristics of the population included in the analysis are presented in Table 1. Additional file 1: Section S3, Fig. S2 shows the age distribution of carriers and non-carriers of APOE $\varepsilon 4$. During follow-up of these individuals, 7460 subsequent visits with clinical diagnosis following a previous diagnosis have been recorded (Table 2). Here, we present and focus on the results derived when utilising the entire sample independently of amyloid status. The change in the transition probabilities and expected time to ADem in amyloid-positive individuals, and the estimated biomarker trajectories in this group, are discussed in Additional file 1: Section S8. In addition, although we considered the influence of education and we present the results of its potential impact on the transition probabilities and expected time to ADem (see Additional file 1: Section S4, Fig. S3), due to the minor and, as yet, unclear effect on the biomarker dynamics, all the biomarker trajectories presented in this study have been developed based on the model that does not incorporate education.

The transition probabilities and the expected times to $\mathrm{MCI}$ and ADem onset as a function of age are illustrated in Fig. 2 for carriers and non-carriers of APOE $\varepsilon 4$. It is observed that the probabilities increase almost exponentially with age, yielding a decrease in the required time to reach more severe clinical states. Depending on age and disease stage, the presence of APOE $\varepsilon 4$ increases the likelihood and rate of progression. The time to ADem from the $\mathrm{CN}$ and MCI states is decreased by more than 10 and 5 years, respectively, when individuals aged 80 years or less are carriers of the APOE $\varepsilon 4$ genotype.

\section{Biomarker trajectories}

For all markers, it is observed that there is a great deal of overlap in measurements across the different diagnostic states, i.e. no single marker is able to accurately distinguish between clinical states (Fig. 3-the parameter estimates of all the biomarker trajectory models are provided in Additional file 1: Table S2). Although it is difficult to draw any conclusions at the individual level, at the population level, the overall biomarker changes occurred during disease development are in agreement with those reported in previous studies; CSF $A \beta_{1-42}$ levels are reduced, $\mathrm{p}$-tau and t-tau levels in CSF and amyloid levels in the brain are elevated, and brain atrophy and hypometabolism, as well as cognitive and memory dysfunction, are observed.

Due to the high variability in measurements, a definite sequence of biomarker changes is difficult to clearly define. However, our model suggests that on average, the concentration of $\mathrm{A} \beta_{1-42}$ in CSF demonstrates slow pathologic changes occurring about 23.2 (95\% CI, 18.928.6) years prior to the clinical presentation of ADem 
Table 1 Dataset used. Number and characteristics of the 2014 individuals included in this analysis, by clinical diagnosis at baseline

\begin{tabular}{|c|c|c|c|c|}
\hline & & \multicolumn{3}{|c|}{ Clinical state at baseline } \\
\hline & & $\mathrm{CN}$ & $\mathrm{MCl}$ & ADem \\
\hline \multicolumn{5}{|l|}{ Number of individuals } \\
\hline Total & & 749 & 910 & 355 \\
\hline \multirow[t]{3}{*}{ Chronological age at baseline (years) } & $\leq 70$ & 241 & 308 & 85 \\
\hline & $70<$ age $<80$ & 402 & 423 & 170 \\
\hline & $>80$ & 106 & 179 & 100 \\
\hline \multirow[t]{2}{*}{ Gender } & Women & 413 & 370 & 157 \\
\hline & Men & 336 & 540 & 198 \\
\hline \multirow[t]{2}{*}{ Years of education } & $\leq 16$ & 385 & 537 & 247 \\
\hline & $>16$ & 364 & 373 & 108 \\
\hline \multirow[t]{3}{*}{ Genetic background (APOE ع4) } & Non-carriers of $\varepsilon 4$ allele & 370 & 420 & 114 \\
\hline & Carriers of $1 \varepsilon 4$ allele & 135 & 337 & 159 \\
\hline & Carriers of $2 \varepsilon 4$ alleles & 12 & 92 & 65 \\
\hline \multicolumn{5}{|c|}{ Other characteristics (standard deviation of the mean in brackets) } \\
\hline \multicolumn{2}{|l|}{ Average age at baseline (years) } & $73.20(6.17)$ & $73.04(7.62)$ & $75.03(7.88)$ \\
\hline \multicolumn{2}{|l|}{ Average number of years of education } & $16.55(2.57)$ & $15.93(2.82)$ & $15.24(2.99)$ \\
\hline \multicolumn{2}{|l|}{ Average time between visits (years) } & $1.02(0.58)$ & $0.8(0.38)$ & $0.64(0.27)$ \\
\hline \multicolumn{2}{|l|}{ Average follow-up (years) } & $3.36(3.43)$ & $3.69(2.62)$ & $1.38(0.85)$ \\
\hline
\end{tabular}

(Figs. 3, 4 and 5). The rate of change peaks much later, approximately 3.6 (95\% CI, 0.9-7.7) years before the ADem clinical symptoms onset, and is followed by a slower deposition which tends towards a plateau. Detectable changes in CSF $\mathrm{t}$-tau and $\mathrm{p}$-tau proteins occur later displaying a similar dynamic, approximately 14 years prior to the ADem onset. No significant difference in the time of occurrence was observed $(13.8$ (95\% CI, 11.1-17.8) years for p-tau and 14.2 (95\% CI, 11.4, 18.2) years for t-tau). On average, PET evidence of $A \beta$ accumulation (AV-45-PET) and brain hypometabolism on FDG-PET are also observed around the same time (Fig. 5).

The hippocampus tends to be the first brain structure affected by the disease; we estimate that detectable hippocampal atrophy could occur as early as the first signs of amyloid deposition (18.8 (95\% CI, 13.1-22.8) years before the ADem onset). Changes in other structural neuroimaging markers, including the whole brain volume (11.7 (95\% CI, 7.1-17.3) years before the ADem

Table 2 Number of transitions from one clinical state to another during follow-up

\begin{tabular}{lllll}
\hline & & \multicolumn{3}{l}{ Subsequent clinical diagnosis } \\
\cline { 3 - 5 } & & CN & MCl & ADem \\
\hline Clinical diagnosis & CN & 2310 & 138 & 4 \\
& MCl & 104 & 3112 & 351 \\
& ADem & 0 & 26 & 1415 \\
\hline
\end{tabular}

onset), are likely to occur much later in the course of the disease (Fig. 5).

Cognitive scores may better reflect continuous changes occurring during clinical progression, albeit with high variability (Fig. 3), with notable changes being observed closer to the onset of ADem. Memory dysfunction, indicated by detectable changes in the LM_Delayed and RAVLT_Immediate scores, could be observed even earlier than changes in CSF $\mathrm{A} \beta_{1-42}, 26.5$ (95\% CI, 24.129.5 ) and 25.6 (95\% CI, 21.9-28.3) years, respectively, before the onset of ADem clinical symptoms (Fig. 5 and Additional file 1: Section S6, Fig. S4). It is also observed that these markers worsen almost in parallel with this protein throughout the full progression period of the disease (Fig. 4). Changes in sensitive scores of clinical performance, such as PACC, could occur as early as 20.5 (95\% CI, 15.9-24) years before ADem and could thus serve as indicators of early cognitive decline in preclinical $\mathrm{AD}$ populations. Global cognitive decline, as assessed by CDR-SB and MMSE, and functional abilities, as assessed by FAQ score, occur last, a few years before the ADem onset (7.2 (95\% CI, 3.9-13.6), $4.3(2.8-7.6)$ and 5 (3.2-7.74) years before ADem, respectively, see also Additional file 1: Section S6, Fig. S4 on the whole dataset). However, FAQ changes at a very high rate, with the highest acceleration occurring within 1 year after the ADem onset (0.16 (95\% CI, 0.58-0.63) years after the onset).

Variations in the levels of plasma markers are difficult to discern. Changes in plasma NfL are more pronounced 


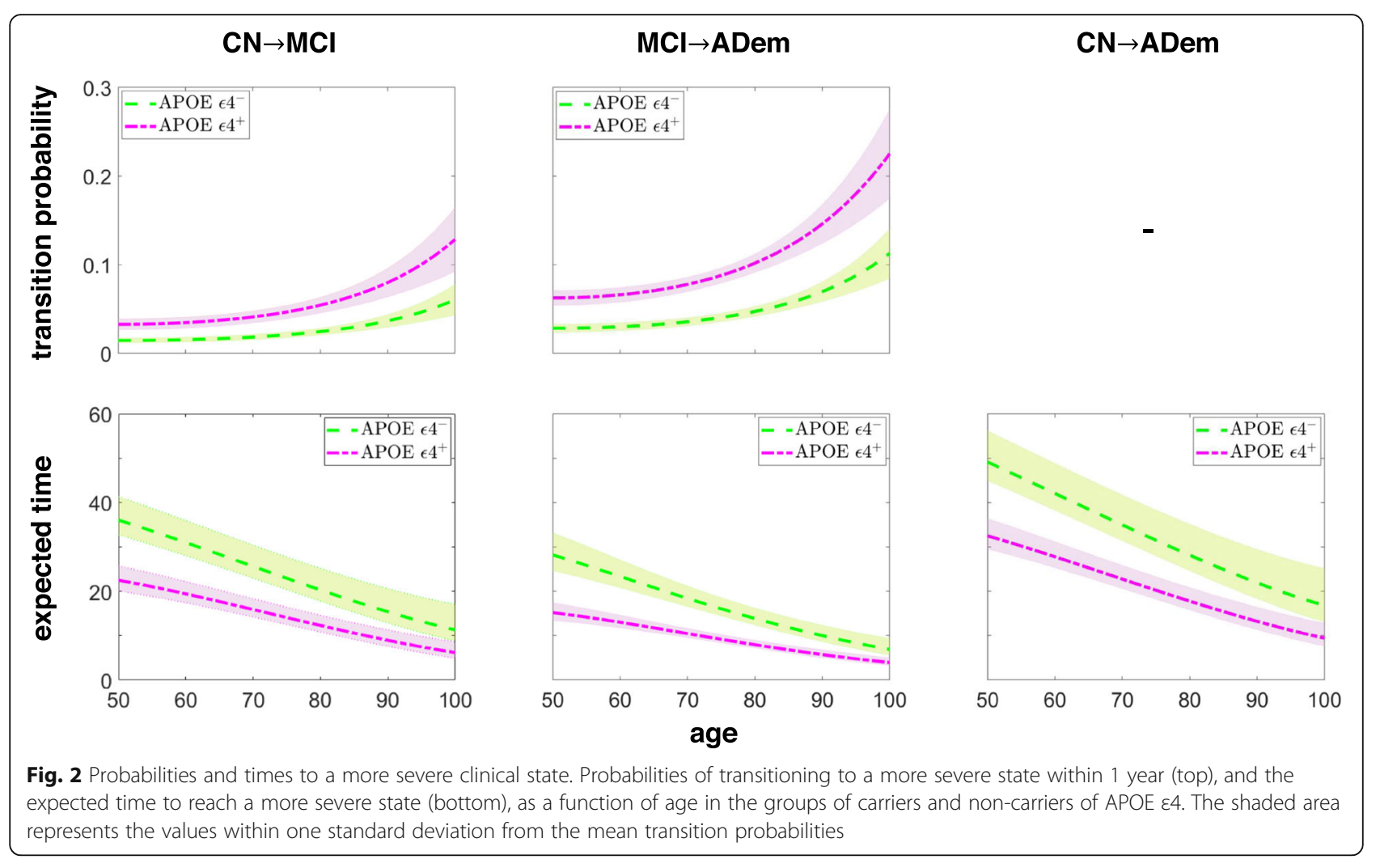

compared to that of the other plasma markers, with the NfL concentration rising at early stages in disease development. Elevated levels of plasma $\mathrm{t}$-tau are observed at more severe clinical states [52].

Figure $5 \mathrm{~b}$ illustrates the time between the inflection point and the ADem onset. This is a good measure in the sense that it can be used to order important changes in the levels of the markers. On average, the highest acceleration of decline in all functional and cognitive abilities, as well as FDG-PET hypometabolism, is expected to take place soon after the clinical manifestation of ADem, following cerebral atrophy, as assessed by the MRI brain volumetric measures. CSF markers, memory impairment (as assessed by LM_Delayed score) and AV-45 PET, which is one of the most direct measures of $A \beta$ accumulation in the brain, could exhibit the highest rate of change much before the ADem onset $[6,13]$ (Fig. 5 and Additional file 1: Section S6, Fig. S4).

\section{Discussion}

In this study, we aimed to characterise the continuous pathophysiological changes that may be observed across the clinical spectrum of $\mathrm{AD}$ and facilitate the interpretation of such changes in clinical settings. We first developed a mathematical model with a probabilistic structure to estimate the expected time interval for each individual from the time of measurement to the onset of a particular clinical state, focusing on the onset of ADem. We then employed these estimates to align short-term individual measurements of cognitive and biological markers on a clinical progression timeline and model their long-term temporal dynamics at the population level. It should be emphasised that although age is one of the important factors affecting the time to a clinical state, individuals are not aligned based on their age, and, despite the high variability, the overall biomarker trajectory can show changes that can occur across the clinical progression to ADem, independently of ageing.

It was shown that the probability of moving to a more severe clinical state increases almost exponentially with age. Depending on the age and clinical state, both the presence of an APOE $\varepsilon 4$ allele and lower levels of education increase the likelihood and rate of developing symptoms of ADem, but the presence of APOE $\varepsilon 4$ was shown to be the dominant factor (it is worth noting that studies have shown that APOE $\varepsilon 4$ is linked to CSF $A \beta_{1-42}$ accumulation $[8,53])$. However, since ADNI is a sample of highly educated people (mean 16 years), the actual influence of education on the clinical expression of AD is unclear in this study and should be interpreted with caution. Many previous studies have shown an association between ADem onset and educational attainment, which could be partly attributed to resistance to cognitive decline due to cognitive reserve, literacy abilities, socioeconomic status, engagement in cognitively 


\section{Cognitive markers}
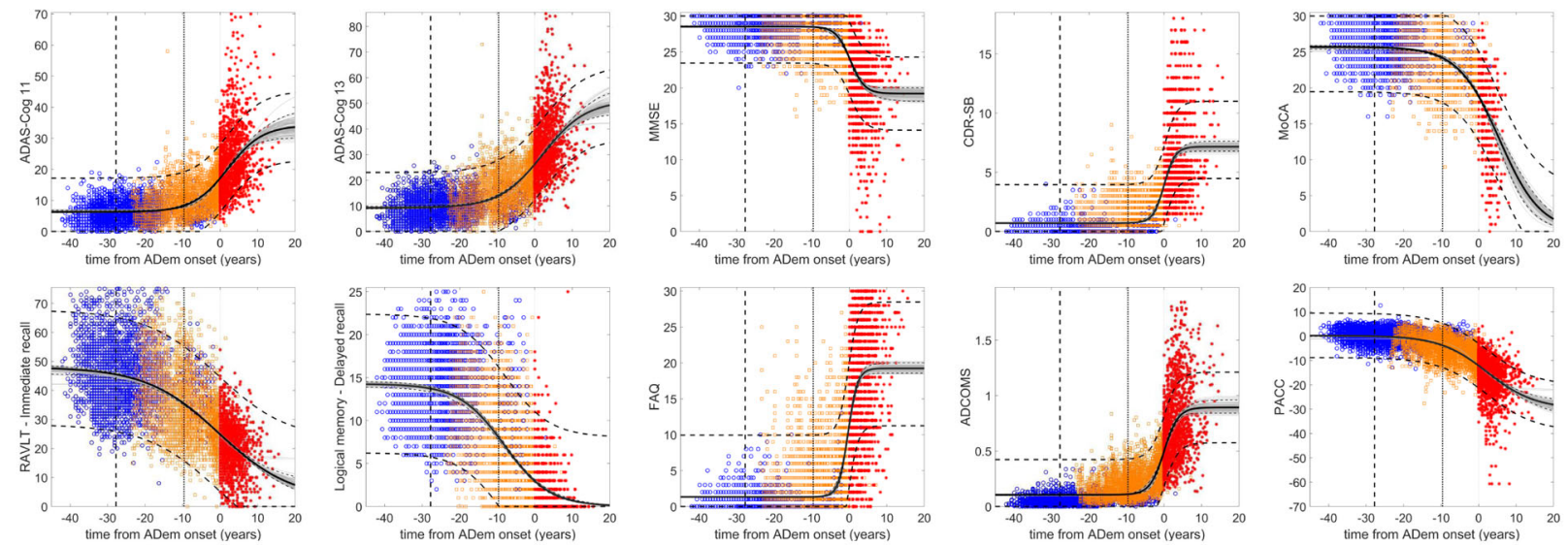

\section{CSF markers}
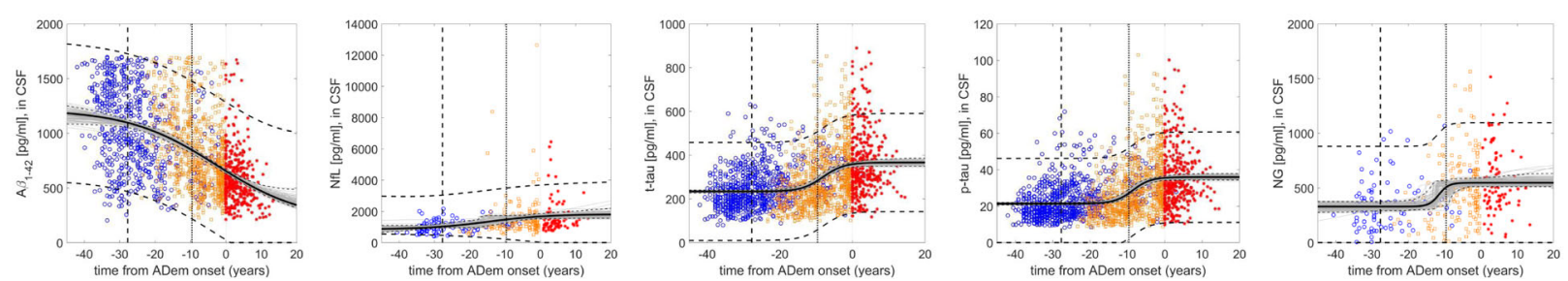

\section{Plasma markers}
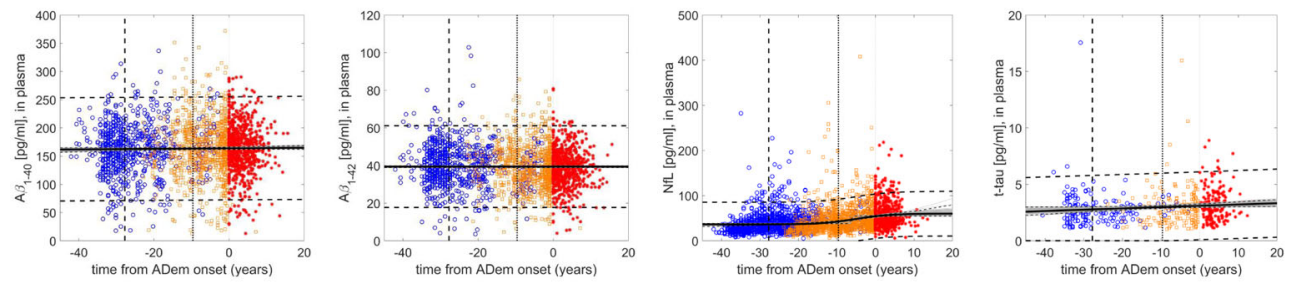

\section{Neuroimaging markers}
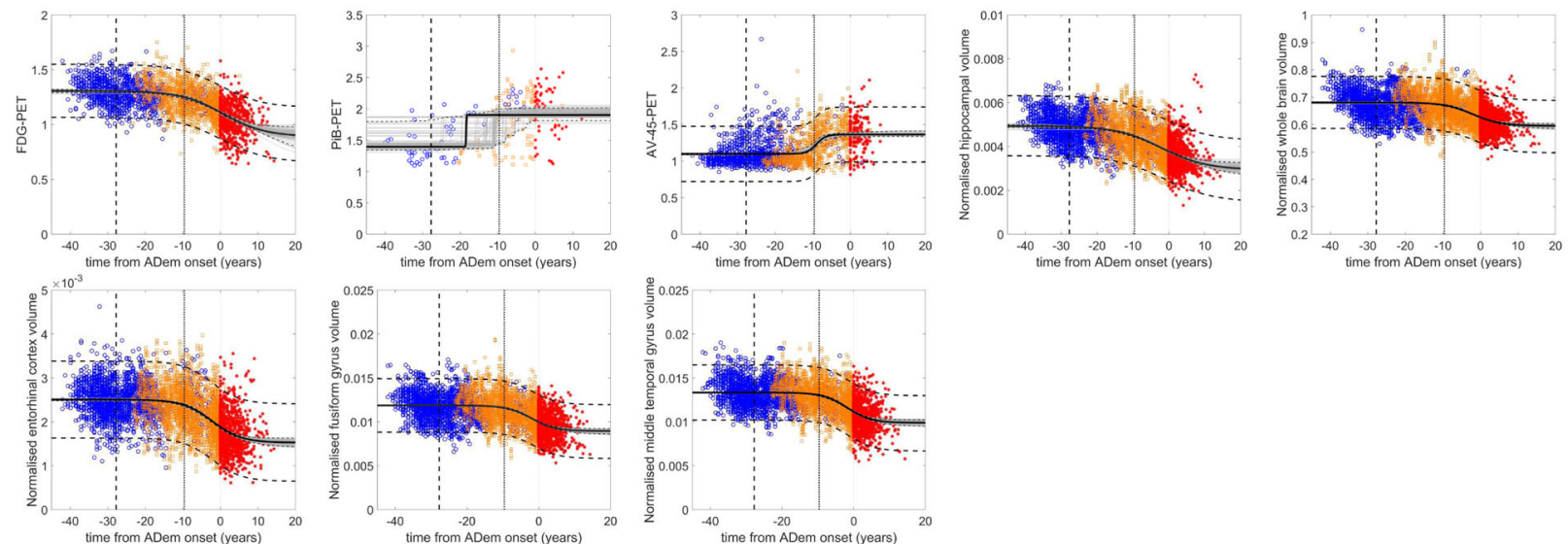

Fig. 3 Trajectories of cognitive, CSF, plasma and neuroimaging markers. The black solid line represents the best fit of the sigmoid function (linear for $A \beta_{1-}$ ${ }_{40}, A \beta_{1-42}$ and t-tau in plasma), the dashed thick lines is the 95\% prediction interval and the dashed thin lines are the estimated $95 \%$ bootstrap $\mathrm{Cl}$ (the 2.5th and 97.5th percentiles for each time point). The light grey lines are the best fit of the function to 100 randomly selected bootstrap samples (out of the 500). The symbols show individual measurements (blue (circles), CN; orange (squares), MCl; red (asterisks), ADem). The vertical dashed and thick dotted lines represent the average expected time from ADem onset of individuals that are CN (27.75 years) and MCl (9.63 years) at baseline, respectively 


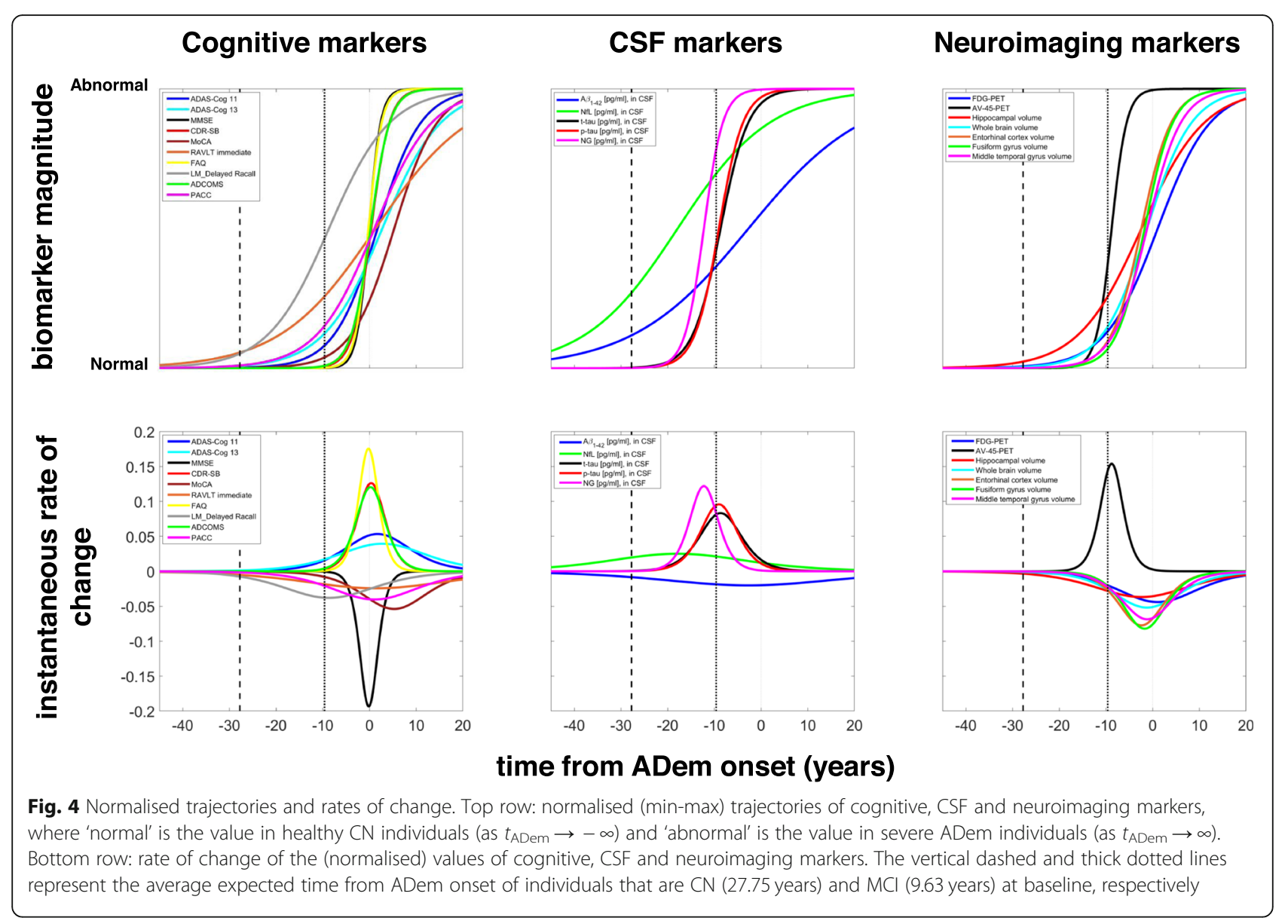

\section{a.}

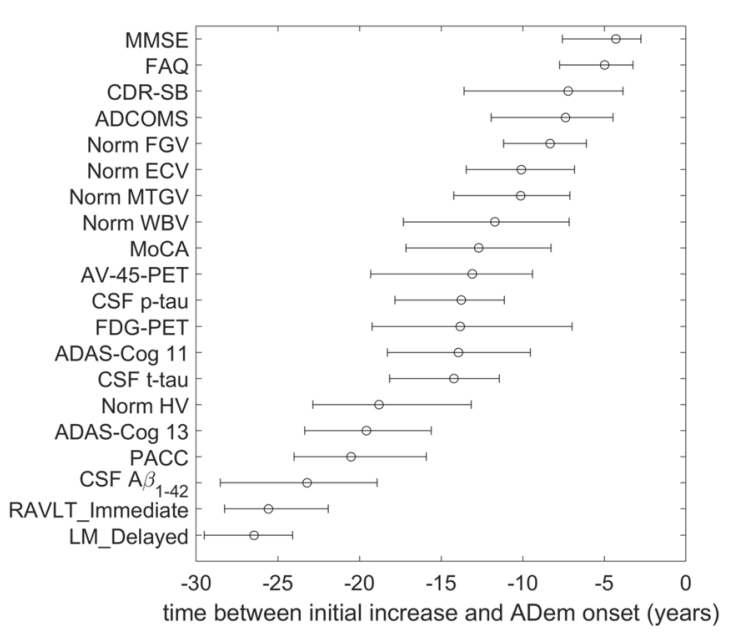

b.

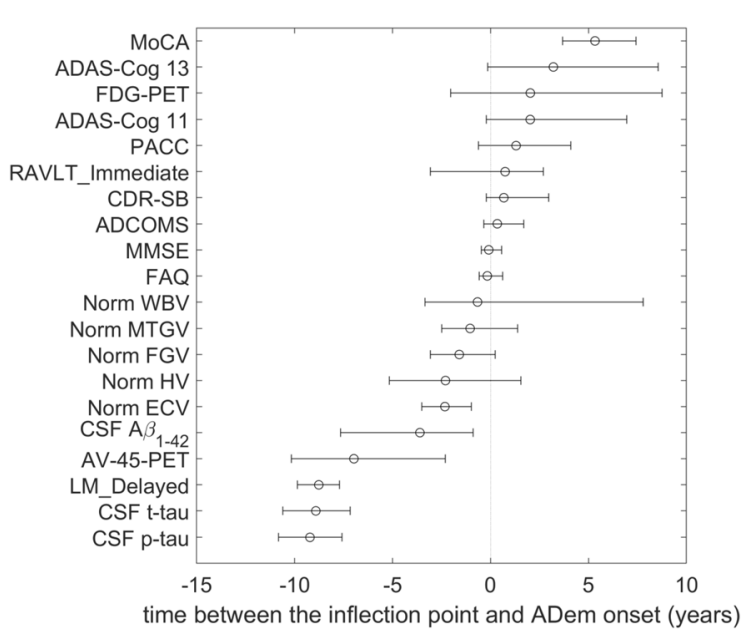

Fig. 5 Initial biomarker changes and inflection points. a The estimated time of the initial change of each of the cognitive, CSF and neuroimaging markers. $\mathbf{b}$ The time at which the inflection point is reached. Error bars show the $95 \% \mathrm{Cl}$ for the mean, estimated by calculating the 2.5 th and 97.5th percentiles of the outputs obtained from 500 bootstrap samples. Only the visits at which CSF A $\beta_{1-42}$ was measured have been included in the analysis. The plasma markers, PiB-PET and CSF NfL and Ng, have been excluded. Norm HV, normalised hippocampal volume; Norm ECV, normalised entorhinal cortex volume; Norm FGV, normalised fusiform gyrus volume; Norm MTGV, normalised middle temporal gyrus volume; Norm WBV, normalised whole brain volume 
demanding work, social activities or occupational roles. This association could also be partly due to a bias in detecting ADem symptoms [54]. However, there are divergent views amongst researchers on the actual impact of education on the development of $\mathrm{AD}$ and the rate of progression $[20,55,56]$.

The results support and contribute to the current understanding of the quantitative characteristics of the clinical progression of AD based on multiple clinical and biological measures $[2,26]$. The timespan and sequence of biomarker changes are also in general agreement with observations in autosomal dominant $\mathrm{AD}[6,57]$. In this study, we estimated and reported the whole time span between the first detectable dynamic biomarker changes and the onset of ADem clinical symptoms. On average, first signs of memory dysfunction and amyloid deposition, as reflected by the levels of $A \beta_{1-42}$ in CSF, could be detected at the pre-symptomatic stage up to about three decades before the occurrence of substantial cognitive and functional decline, which are mainly observed at early stages of ADem [2]. Both markers also seem to change at a similar rate during the disease progression. This may imply a link between memory deficits and elevated amyloid deposition, a view that is supported by previous reports $[2,58-61]$. The value of episodic memory tests, and associations of changes in the performance in delayed recall tasks and the clinical progression to ADem, has also been observed and discussed in previous studies [2, 9, 10, 62-65]. However, it should be noted that such markers may not be sufficient for the improvement of participant recruitment and endpoint choice in very early-stage prevention trials. Even if first CSF $A \beta_{1-}$ ${ }_{42}$ changes and disease-associated subtle memory deficits could be detected very early at the preclinical stage, the short time window within which substantial changes occur and the fact that individuals can have amyloid pathology for a long period without development of substantial cognitive dysfunction and brain pathology, as shown in several preclinical studies $[8,61]$, demonstrate the difficulties of recruiting individuals at the preclinical stage of $\mathrm{AD}$ and present obvious challenges in the design of clinical trials of preventative treatments.

An interesting result derived from our analyses is the suggestion that changes in CSF NfL [40], a biomarker of neuronal damage, could also occur approximately over the same timescale as memory decline and $A \beta_{1-42}$ accumulation in CSF. However, more data is required to support this suggested pattern. Progressing towards ADem, signs of memory dysfunction and changes in CSF $A \beta_{1-42}$ are closely followed by first signs of cognitive decline, as assessed by sensitive cognitive performance measures (e.g. PACC), and alterations in brain structure measured by hippocampal atrophy. The value of such markers, and in particular the ability of $A \beta_{1-42}$ in CSF, cognitive (free recall) and normalised hippocampal volume to predict the onset of symptomatic sporadic $\mathrm{AD}$ (time to $\mathrm{CDR}>$ 0 ), has also been reported in a recent study [8]. Estimated changes in other CSF measures such as t-tau and p-tau, brain structural measures and brain glucose metabolism are observed later. Loss of functional abilities occurs last, closer to the ADem onset. Significant atrophy in brain regions, such as the entorhinal cortex, fusiform gyrus and middle temporal gyrus, occurs after initial hippocampal atrophy. However, at later stages, their expected atrophy rate becomes higher than that of the hippocampus and whole brain, and the estimates of the time point at which the maximum rate occurs are relatively precise, which may be attributed to the smaller size of these structures. Hence, such brain regions may be more sensitive biomarkers to changes than bigger brain regions [66]. Significant volume loss in all brain regions is likely to be observed just before the clinical diagnosis of ADem and rapid decline in cognitive abilities, which may indicate an association.

The accurate measurement of proteins in the blood and identification of a clear association with brain pathology are still challenging, potentially due to the effect of factors that are not directly related to the disease stage of individuals, including clearance mechanisms and high levels of these proteins produced in the peripheral organs. However, the ease and low cost of measurements have created much interest in identifying reliable and sensitive peripheral blood-based markers that could serve as biomarkers of $\mathrm{AD}$ [67]. The changes in the plasma levels of proteins considered in this study were not as evident as those of other markers measured in CSF and the brain. However, plasma NfL seems to be a promising marker that can indicate pathological changes at early stages in disease development, and therefore, although not specific for $\mathrm{AD}$, it could potentially be considered as a biomarker for the screening of related neurodegeneration and disease prognosis, monitoring and assessment [68-71].

The rate of change and sensitivity of the various markers varies across the different stages of the $\mathrm{AD}$ continuum [26], and so, the order in which they reach specific degrees of abnormality may differ. This, perhaps, suggests that the appropriateness of each marker for disease diagnosis and monitoring and use in clinical trials should be based on the disease stage $[8,72]$. Considering the rate of change of potential markers instead of just a single absolute measure at a specific point in time may help identify the stage in disease progression [8]. For some markers, such as t-tau and p-tau, the results suggest that the average rate of change tends to zero around the occurrence of clinical ADem symptoms. This may be an implication of severe and irreversible pathological changes that may happen well before individuals reach 
severe clinical stages. On the other hand, some biomarkers, such as neuroimaging markers, remain dynamic even after onset, which may imply that there might be late stages where therapeutic interventions can be effective. Such late biomarkers may also be more useful in monitoring disease progression at severe disease stages [13]. In this study, it is shown that substantial dynamic changes in $A \beta_{1-42}$ are also expected to continue to occur after the clinical presentation of ADem. This contradicts previous studies in preclinical Alzheimer's disease which suggested that $A \beta_{1-42}$ reaches a plateau by the time of ADem onset [61, 72]. However, the late disease stages are not well represented in the ADNI sample which may have biassed the estimation of the late biomarker behaviour. Thus, whether biomarkers reach a plateau or continue to decelerate after ADem onset needs to be validated when more longitudinal data at late stages become available.

In addition to modelling the biomarker trajectories using all available ADNI data, the trajectories have also been estimated in a sample of individuals that may be more likely to be in the Alzheimer's continuum due to the evidence of brain $A \beta$ accumulation observed during the study [16]. It has been shown that, on average, individuals in the amyloid-positive group tend to move faster towards more severe clinical states, potentially because they are at an advanced stage in disease development. However, no remarkable differences were observed in the average qualitative and quantitative behaviour of biomarkers throughout the disease progression when compared to the biomarkers estimated in the entire sample, especially in the late disease stages. This may be attributed to the fact that ADNI participants are a highly selective group who have an increased risk of developing the disease (almost $50 \%$ of the individuals are classified in the amyloid-positive group). Differences, however, may be pronounced when comparing the trajectories during follow-up in the study between the groups of those that have and those that have not shown elevated levels of $A \beta$ at baseline [37].

It is worth emphasising that whether biomarker changes as predicted by the models can be detected in a clinical study is uncertain. The high variability and overlap between the measurements at the different clinical states confirm difficulties in detecting significant biomarker changes and in making accurate predictions at the individual level. Hence, the order at which changes in biomarkers can be detected might be different from the current general view of biomarker change patterns [12]. In addition, due to the lack of knowledge of the exact impact of each biomarker change on the biological process of disease development, it is difficult to assess their importance based on the changes observed, as a small change in one marker might be more important than a large change in another marker.
The model estimations are contingent on the validity of a series of simple assumptions. The mathematical model assumes that at every given state, the future of the system (for example, the probability of progressing to ADem in the next year given the current state is $\mathrm{MCI}$ ) is entirely independent of the history of the system (for example, at which states individuals were before and for how long). However, this limitation is partly mitigated by the fact that transition probabilities in the model depend on the current diagnostic state and age, and thus, depending on the clinical state, older individuals are more likely to transition to more severe states independently of the system's history. In addition, the model relies on pre-defined distinct clinical states and the accuracy of clinical diagnosis. Despite the high measurement noise in clinical scores and the high rate of clinical misdiagnosis [22], it was also assumed that individuals transitioned to a different clinical state at the visit when the diagnostic criteria were satisfied, even though, due to the long-term/gradual nature of $\mathrm{AD}$ progression and the sequential change in different pathophysiological processes, defining any other distinct boundaries between the states is challenging. The current categorisation of individuals in clinical groups is also based on a simplified set of criteria, which does not fully represent their biomarker profile and does not take into account various sources of uncertainty. Hence, although this classification is useful in clinical practice, it increases heterogeneity within and overlap between the biomarker measurements in the clinical groups. Indeed, the overlap for some biomarkers could also be partly attributed to neurobiological changes occurring in nonAlzheimer's ageing population, due for example to ageing or the presence of other pathological processes and neurodegenerative or cardiovascular diseases, that can be present in all clinical stages [73].

As a biomarker model, and based on the current understanding of the pathophysiological processes that are associated with $\mathrm{AD}$ [26], a sigmoid function of time was chosen to describe biomarker temporal dynamics. Despite the high variability and the limited data to support very early and late stages in disease progression, this choice was supported by the available data and it described quite well the dynamic behaviour of most of the biomarkers. It also allowed us to estimate when significant biomarker changes occur, as well as the point at which biomarkers are most likely to reach the maximum rate of change before they potentially begin to plateau. In addition, it was assumed that all individuals will follow the same pathway of neuropathological changes towards ADem. Although the sample of individuals considered are at high risk of developing ADem, this may have resulted in the overestimation of the expected times to a clinical state, which may have in turn 
influenced the estimation of the biomarker dynamics and the point at which first significant changes occur.

Although all biomarker data has been synchronised on the same disease progression timeline independently of the levels of any of the biomarkers, estimating the trajectories utilising a complete dataset for all biomarkers would enable us to make a better comparison between the biomarker dynamics, and in particular the point at which the first significant change and the inflection point occur. Due to the limited data, a complete dataset could not support the model of changes in many biomarkers and the estimation of the quantities considered. When utilising the whole dataset, the results produced were more precise for biomarkers with more measurements, even though in this case many biomarkers may not be comparable. However, in any case, a definite sequence of pathophysiological events that lead to ADem cannot yet be suggested given the available data. More frequent measurements taken systematically at the same time for all biomarkers would enable us to reduce data variability and produce more precise results. Increasing the sample size, reducing data heterogeneity and defining the error of each measurement/assay would greatly facilitate the detection of even earlier changes than the ones presented in the current study.

The model can be used to estimate the biomarker trajectories in different risk groups. It can also be extended to incorporate other potential confounding variables, such as the level of specific biomarkers; so, given the characteristics of an individual and the levels of biomarkers included in the model, the expected time to ADem, or other disease states, will be estimated. The same methodology can also be applied to describe the sequence and magnitude of biomarker changes with respect to the time required to reach a specific biomarker value, e.g. a defined biomarker threshold/cut-off point $[8,74]$. The estimation of these times could help in the identification of individuals at the earliest disease stages that could be recruited in secondary prevention trials, where, currently, participants are included if they have already developed a biomarker pathology defined by conventional biomarker thresholds.

\section{Conclusions}

The model presented here estimates the continuous changes that can be observed in the level of cognitive and biological markers during the clinical progression of cognitively unimpaired individuals to Alzheimer's clinical syndrome. Despite the high variability within and between individuals at the different clinical states, it demonstrates the long period of asynchronous pathologic changes occurring along with the clinical progression and supports and complements previous conceptual and hypothetical models on the pathophysiological cascade [26]; amyloid deposition and signs of memory decline can occur in cognitively unimpaired individuals as early as 30 years prior to the onset of Alzheimer's clinical syndrome. A sequence of events, including tauopathy, structural brain changes and neurodegeneration, follows before the onset of severe cognitive symptoms. Such models can provide insights into the underlying causes of the clinical symptoms and support the choice of appropriate endpoints in clinical trials according to the stage in disease progression. They can also facilitate the identification of the position of individuals in the pathophysiological continuum, which will be an important tool for improving the recruitment process in clinical trials and the assessment of treatment outcomes.

\section{Supplementary information}

Supplementary information accompanies this paper at https://doi.org/10. 1186/s13195-020-00636-z.

Additional file 1: Supplementary information, tables and figures.

\section{Abbreviations}

A $\beta$ : Amyloid- $\beta$; ADAS-Cog: Alzheimer's Disease Assessment Scale-Cognitive Subscale; ADCOMS: Alzheimer's Disease Composite Score; ADNI: Alzheimer's Disease Neuroimaging Initiative; APOE: Apolipoprotein E; CDR-SB: Clinical Dementia Rating Scale-Sum of Boxes; CN: Cognitively normal; F18-AV45: Florbetapir; FAQ: Functional Activities Questionnaire; FDG: ${ }^{18} \mathrm{~F}-$ fluorodeoxyglucose; ICV: Intracranial volume; $\mathrm{MCl}$ : Mild cognitive impairment; MMSE: Mini-Mental State Examination; MoCA: Montreal Cognitive Assessment; NfL: Neurofilament light; Ng: Neurogranin; PACC: Preclinical Alzheimer Cognitive Composite; PiB: Pittsburgh compound B; $\mathrm{p}$ tau: Phosphorylated tau; RAVLT: Rey's Auditory Verbal Learning Test; SUVr: Standardised uptake value ratio; t-tau: Total tau; LM_Delayed: Logical Memory-Delayed recall

\section{Acknowledgements}

We would like to thank three anonymous reviewers whose valuable comments have helped us to significantly improve the quality of this study.

This study was funded by the Medical Research Council (MRC) and Dementias Platform UK (DPUK)—grant reference MR/R015600/1. DPUK provided the infrastructure for this project through MRC grant ref. MR/ L023784/2. We acknowledge joint Centre funding from the UK Medical Research Council and the Department for International Development through grant ref. MR/R015600/1.

Data collection and sharing for this project was funded by the Alzheimer's Disease Neuroimaging Initiative (ADNI) (National Institutes of Health Grant U01 AG024904) and DOD ADNI (Department of Defense award number W81XWH-12-2-0012). The ADNI was launched in 2003 as a public-private partnership, led by principal investigator Michael W. Weiner, MD. The primary goal of ADNI has been to test whether serial magnetic resonance imaging $(\mathrm{MRI})$, positron emission tomography (PET), other biological markers and clinical and neuropsychological assessment can be combined to measure the progression of mild cognitive impairment $(\mathrm{MCl})$ and early Alzheimer's disease (AD). For up-to-date information, see www.adni-info.org.

ADNI is funded by the National Institute on Aging, the National Institute of

Biomedical Imaging and Bioengineering, and through generous contributions from the following: AbbVie, Alzheimer's Association; Alzheimer's Drug Discovery Foundation; Araclon Biotech; BioClinica, Inc.; Biogen; Bristol-Myers Squibb Company; CereSpir, Inc.; Cogstate; Eisai Inc.; Elan Pharmaceuticals, Inc.; Eli Lilly and Company; Eurolmmun; F. Hoffmann-La Roche Ltd. and its affiliated company Genentech, Inc.; Fujirebio; GE Healthcare; IXICO Ltd.; Janssen Alzheimer Immunotherapy Research \& Development, LLC.; Johnson \& Johnson Pharmaceutical Research \& Development LLC.; Lumosity; Lundbeck; Merck \& Co., Inc.; Meso Scale Diagnostics, LLC.; NeuroRx Research; Neurotrack Technologies; Novartis Pharmaceuticals 
Corporation; Pfizer Inc.; Piramal Imaging; Servier; Takeda Pharmaceutical Company; and Transition Therapeutics. The Canadian Institutes of Health Research is providing funds to support ADNI clinical sites in Canada. Private sector contributions are facilitated by the Foundation for the National Institutes of Health (www.fnih.org). The grantee organisation is the Northern California Institute for Research and Education, and the study is coordinated by the Alzheimer's Therapeutic Research Institute at the University of Southern California. ADNI data are disseminated by the Laboratory for Neuro Imaging at the University of Southern California.

Data used in the preparation of this article were obtained from the Alzheimer's Disease Neuroimaging Initiative (ADNI) database (adni.loni.usc. edu). As such, the investigators within the ADNI contributed to the design and implementation of ADNI and/or provided data but did not participate in the analysis or writing of this report. A complete listing of ADNI investigators can be found at http://adni.loni.usc.edu/wp-content/uploads/how_to_apply/ ADNI_Acknowledgement_List.pdf.

\section{Authors' contributions}

$\mathrm{CH}$ designed the research and wrote the manuscript. $\mathrm{CH}$ and SE performed the research. $C H, S E, S B, L C S$ and KMM analysed and interpreted the data. $\mathrm{CH}, \mathrm{SE}, \mathrm{SB}, \mathrm{LCS}, \mathrm{KMM}, \mathrm{FdW}$ and RMA reviewed and revised the paper. All authors read and approved the final manuscript.

\section{Funding}

This study was funded by the Medical Research Council (MRC) and Dementias Platform UK (DPUK) — grant reference MR/R015600/1. The funding body had no role in the design of the study and collection, analysis, interpretation of data, and writing of the manuscript.

\section{Availability of data and materials}

The datasets generated and analysed during the current study are available in the ADNI data repository, http://adni.loni.usc.edu/data-samples/accessdata/.

\section{Ethics approval and consent to participate}

The study procedures were approved by the institutional review boards of all participating centres (https://adni.loni.usc.edu/wp-content/uploads/how_ to_apply/ADNI_Acknowledgement_List.pdf), and written informed consent was obtained from all participants or their authorised representatives. Ethics approval was obtained from the institutional review boards of each institution involved: Oregon Health and Science University; University of Southern California; University of California—San Diego; University of Michigan; Mayo Clinic, Rochester; Baylor College of Medicine; Columbia University Medical Center; Washington University, St. Louis; University of Alabama at Birmingham; Mount Sinai School of Medicine; Rush University Medical Center; Wien Center; Johns Hopkins University; New York University; Duke University Medical Center; University of Pennsylvania; University of Kentucky; University of Pittsburgh; University of Rochester Medical Center; University of California, Irvine; University of Texas Southwestern Medical School; Emory University; University of Kansas, Medical Center; University of California, Los Angeles; Mayo Clinic, Jacksonville; Indiana University; Yale University School of Medicine; McGill University, Montreal-Jewish General Hospital; Sunnybrook Health Sciences, Ontario; U.B.C. Clinic for AD \& Related Disorders; Cognitive Neurology — St. Joseph's, Ontario; Cleveland Clinic Lou Ruvo Center for Brain Health; Northwestern University; Premiere Research Inst (Palm Beach Neurology); Georgetown University Medical Center; Brigham and Women's Hospital; Stanford University; Banner Sun Health Research Institute; Boston University; Howard University; Case Western Reserve University; University of California, Davis—Sacramento; Neurological Care of CNY; Parkwood Hospital; University of Wisconsin; University of California, Irvine-BIC; Banner Alzheimer's Institute; Dent Neurologic Institute; Ohio State University; Albany Medical College; Hartford Hospital, Olin Neuropsychiatry Research Center; Dartmouth-Hitchcock Medical Center; Wake Forest University Health Sciences; Rhode Island Hospital; Butler Hospital; UC San Francisco; Medical University South Carolina; St. Joseph's Health Care Nathan Kline Institute; University of lowa College of Medicine; and Cornell University and University of South Florida: USF Health Byrd Alzheimer's Institute. The investigators within the ADNI contributed to the design and implementation of the ADNI and/or provided data but did not participate in the analysis or writing of this report. A complete listing of ADNI investigators can be found online (http://adni.
loni.usc.edu/wp-content/uploads/how_to_apply/ADNI_Acknowledgement_ List.pdf).

\section{Consent for publication}

Not applicable.

\section{Competing interests}

RMA is a non-executive board member of GlaxoSmithKline (GSK). GSK played no part in this research, its funding or the preparation of the manuscript.

\section{Author details}

${ }^{1}$ Department of Infectious Disease Epidemiology, School of Public Health Imperial College London, London, UK. ${ }^{2}$ Modelling and Economics Unit, National Infection Service, Public Health England, London, UK. ${ }^{3}$ Lysholm Department of Neuroradiology, National Hospital for Neurology and Neurosurgery, University College London Hospitals, London, UK.

Received: 13 November 2019 Accepted: 20 May 2020

Published online: 13 June 2020

\section{References}

1. Jack CR Jr, Albert MS, Knopman DS, McKhann GM, Sperling RA, Carrillo MC, Thies B, Phelps $\mathrm{CH}$. Introduction to the recommendations from the National Institute on Aging-Alzheimer's Association workgroups on diagnostic guidelines for Alzheimer's disease. Alzheimers Dement. 2011;7:257-62.

2. Villemagne $V L$, Burnham $S$, Bourgeat $P$, Brown B, Ellis KA, Salvado O, Szoeke C, Macaulay SL, Martins R, Maruff $P$, et al. Amyloid $\beta$ deposition, neurodegeneration, and cognitive decline in sporadic Alzheimer's disease: a prospective cohort study. Lancet Neurol. 2013;12:357-67.

3. Aisen PS, Cummings J, Jack CR, Morris JC, Sperling R, Frölich L, Jones RW, Dowsett SA, Matthews BR, Raskin J, et al. On the path to 2025: understanding the Alzheimer's disease continuum. Alzheimers Res Ther. 2017:9:60.

4. Lo RY, Hubbard AE, Shaw LM, Trojanowski JQ, Petersen RC, Aisen PS, Weiner MW, Jagust WJ. Alzheimer's disease neuroimaging I: longitudinal change of biomarkers in cognitive decline. Arch Neurol. 2011;68:1257-66.

5. Petersen RC, Aisen PS, Beckett LA, Donohue MC, Gamst AC, Harvey DJ, Jack CR Jr, Jagust WJ, Shaw LM, Toga AW, et al. Alzheimer's Disease Neuroimaging Initiative (ADNI): clinical characterization. Neurology. 2010;74: 201-9.

6. Bateman RJ, Xiong C, Benzinger TLS, Fagan AM, Goate A, Fox NC, Marcus DS, Cairns NJ, Xie X, Blazey TM, et al. Clinical and biomarker changes in dominantly inherited Alzheimer's disease. N Engl J Med. 2012;367:795-804.

7. Ryman DC, Acosta-Baena N, Aisen PS, Bird T, Danek A, Fox NC, Goate A, Frommelt $P$, Ghetti B, Langbaum JBS, et al. Symptom onset in autosomal dominant Alzheimer disease: a systematic review and meta-analysis. Neurology. 2014;83:253-60.

8. Roe CM, Ances BM, Head D, Babulal GM, Stout SH, Grant EA, Hassenstab J, Xiong C, Holtzman DM, Benzinger TLS, et al. Incident cognitive impairment: longitudinal changes in molecular, structural and cognitive biomarkers. Brain. 2018;141:3233-48.

9. Jedynak BM, Lang A, Liu B, Katz E, Zhang Y, Wyman BT, Raunig D, Jedynak CP, Caffo B, Prince JL, Alzheimer's Disease Neuroimaging I. A computational neurodegenerative disease progression score: method and results with the Alzheimer's disease neuroimaging initiative cohort. Neurolmage. 2012;63: 1478-86.

10. Bilgel $M$, Jedynak BM. Predicting time to dementia using a quantitative template of disease progression. Alzheimer's Dementia. 2019;11:205-15.

11. Li D, Iddi S, Thompson WK, Donohue MC. Bayesian latent time joint mixed effect models for multicohort longitudinal data. Stat Methods Med Res. 2017:28:835-45.

12. Donohue MC, Jacqmin-Gadda H, Le Goff M, Thomas RG, Raman R, Gamst AC, Beckett LA, Jack CR Jr, Weiner MW, Dartigues J-F, et al. Estimating longterm multivariate progression from short-term data. Alzheimer's Dementia. 2014;10:S400-10.

13. Oxtoby NP, Young AL, Cash DM, Benzinger TLS, Fagan AM, Morris JC, Bateman RJ, Fox NC, Schott JM, Alexander DC. Data-driven models of dominantly-inherited Alzheimer's disease progression. Brain. 2018;141:1529_ 44. 
14. Young AL, Oxtoby NP, Ourselin S, Schott JM, Alexander DC. A simulation system for biomarker evolution in neurodegenerative disease. Med Image Anal. 2015;26:47-56.

15. Yang E, Farnum M, Lobanov V, Schultz T, Verbeeck R, Raghavan N, Samtani MN, Novak G, Narayan V, DiBernardo A. Quantifying the pathophysiological timeline of Alzheimer's disease. J Alzheimers Dis. 2011;26:745-53.

16. Jack CR Jr, Bennett DA, Blennow K, Carrillo MC, Dunn B, Haeberlein SB, Holtzman DM, Jagust W, Jessen F, Karlawish J, et al. NIA-AA Research Framework: toward a biological definition of Alzheimer's disease. Alzheimer's Dementia. 2018;14:535-62.

17. Petersen RC: How early can we diagnose Alzheimer disease (and is it sufficient)? The 2017 Wartenberg lecture 2018, 91:395-402.

18. McKhann G, Drachman D, Folstein M, Katzman R, Price D, Stadlan EM. Clinical diagnosis of Alzheimer's disease: report of the NINCDS-ADRDA Work Group under the auspices of Department of Health and Human Services Task Force on Alzheimer's Disease. Neurology. 1984;34:939-44.

19. Liu CC, Liu CC, Kanekiyo T, Xu H, Bu G. Apolipoprotein E and Alzheimer disease: risk, mechanisms and therapy. Nat Rev Neurol. 2013;9:106-18.

20. Sharp ES, Gatz M. Relationship between education and dementia: an updated systematic review. Alzheimer Dis Assoc Disord. 2011;25:289-304.

21. Hadjichrysanthou C, Ower AK, de Wolf F, Anderson RM, for the Alzheimer's Disease Neuroimaging I. The development of a stochastic mathematical model of Alzheimer's disease to help improve the design of clinical trials of potential treatments. PLoS One. 2018;13:e0190615.

22. Hadjichrysanthou C, McRae-McKee K, Evans S, de Wolf F, Anderson RM. Potential factors associated with cognitive improvement of individuals diagnosed with mild cognitive impairment or dementia in longitudinal studies. J Alzheimers Dis. 2018;66:587-600.

23. Evans S, McRae-McKee K, Hadjichrysanthou C, Wong MM, Ames D, Lopez O, de Wolf F, Anderson RM. Alzheimer's disease progression and risk factors: a standardized comparison between six large data sets. Alzheimer's Dementia. 2019:5:515-23.

24. Bonham LW, Geier EG, Fan CC, Leong JK, Besser L, Kukull WA, Kornak J, Andreassen OA, Schellenberg GD, Rosen $\mathrm{HJ}$, et al. Age-dependent effects of APOE $\varepsilon 4$ in preclinical Alzheimer's disease. Ann Clin Transl Neurol. 2016;3: 668-77.

25. Liu L, Caselli RJ. Age stratification corrects bias in estimated hazard of APOE genotype for Alzheimer's disease. Alzheimer's Dementia. 2018:4:602-8.

26. Jack CR Jr, Knopman DS, Jagust WJ, Shaw LM, Aisen PS, Weiner MW Petersen RC, Trojanowski JQ. Hypothetical model of dynamic biomarkers of the Alzheimer's pathological cascade. Lancet Neurol. 2010;9:119-28.

27. Rosen WG, Mohs RC, Davis KL. A new rating scale for Alzheimer's disease. Am J Psychiatry. 1984;141:1356-64.

28. Mohs RC, Knopman D, Petersen RC, Ferris SH, Ernesto C, Grundman M, Sano M, Bieliauskas L, Geldmacher D, Clark C, Thal LJ. Development of cognitive instruments for use in clinical trials of antidementia drugs: additions to the Alzheimer's Disease Assessment Scale that broaden its scope. The Alzheimer's Disease Cooperative Study. Alzheimer Dis Assoc Disord. 1997; 11(Suppl 2):S13-21.

29. Folstein MF, Folstein SE, PR MH. "Mini-Mental State": a practical method for grading the cognitive state of patients for the clinician. J Psychiatr Res. 1975;12:189-98.

30. Hughes CP, Berg L, Danziger WL, Coben LA, Martin RL. A new clinical scale for the staging of dementia. Br J Psychiatry. 1982;140:566-72.

31. Berg L, Miller JP, Storandt M, Duchek J, Morris JC, Rubin EH, Burke WJ, Coben LA. Mild senile dementia of the Alzheimer type: 2. Longitudinal assessment. Ann Neurol. 1988;23:477-84.

32. Nasreddine ZS, Phillips NA, Bedirian V, Charbonneau S, Whitehead V, Collin I, Cummings JL, Chertkow H. The Montreal Cognitive Assessment, MoCA: a brief screening tool for mild cognitive impairment. J Am Geriatr Soc. 2005; 53:695-9.

33. Harrah CH Jr, Chance JM, Pfeffer Rl, Kurosaki TT, Filos S. Measurement of functional activities in older adults in the community. J Gerontol. 1982;37: 323-9.

34. Rey A. L' examen clinique en psychologie. Paris: Presses universitaires de France; 1964

35. Wechsler D: WMS-R: Wechsler Memory Scale-Revised: manual. San Antonio: Psychological Corp.: Harcourt Brace Jovanovich; 1987.

36. Donohue MC, Sperling RA, Salmon DP, Rentz DM, Raman R, Thomas RG, Weiner M, Aisen PS. The preclinical Alzheimer cognitive composite: measuring amyloid-related decline. JAMA Neurol. 2014;71:961-70.
37. Donohue MC, Sperling RA, Petersen R, Sun C-K, Weiner MW, Aisen PS, Initiative ftAsDN. Association between elevated brain amyloid and subsequent cognitive decline among cognitively normal persons. JAMA. 2017:317:2305-16.

38. Wang J, Logovinsky V, Hendrix SB, Stanworth SH, Perdomo C, Xu L, Dhadda S, Do I, Rabe M, Luthman J, et al. ADCOMS: a composite clinical outcome for prodromal Alzheimer's disease trials. J Neurol Neurosurg Psychiatry. 2016;87:993-9.

39. Blennow K, Hampel H. CSF markers for incipient Alzheimer's disease. Lancet Neurol. 2003;2:605-13.

40. Zetterberg H, Skillback T, Mattsson N, Trojanowski JQ, Portelius E, Shaw LM, Weiner MW, Blennow K. Association of cerebrospinal fluid neurofilament light concentration with Alzheimer disease progression. JAMA Neurol. 2016; 73:60-7.

41. Thorsell A, Bjerke M, Gobom J, Brunhage E, Vanmechelen E, Andreasen N, Hansson O, Minthon L, Zetterberg H, Blennow K. Neurogranin in cerebrospinal fluid as a marker of synaptic degeneration in Alzheimer's disease. Brain Res. 2010;1362:13-22.

42. Fox NC, Schott JM. Imaging cerebral atrophy: normal ageing to Alzheimer's disease. Lancet. 2004;363:392-4.

43. Whitwell JL, Crum WR, Watt HC, Fox NC. Normalization of cerebral volumes by use of intracranial volume: implications for longitudinal quantitative MR imaging. Am J Neuroradiol. 2001;22:1483.

44. Foster NL, Heidebrink JL, Clark CM, Jagust WJ, Arnold SE, Barbas NR, DeCarli CS, Scott Turner R, Koeppe RA, Higdon R, Minoshima S. FDG-PET improves accuracy in distinguishing frontotemporal dementia and Alzheimer's disease. Brain. 2007;130:2616-35.

45. Klunk WE, Engler H, Nordberg A, Wang Y, Blomqvist G, Holt DP, Bergstrom M, Savitcheva I, Huang GF, Estrada S, et al. Imaging brain amyloid in Alzheimer's disease with Pittsburgh compound-B. Ann Neurol. 2004;55:306-19.

46. Clark CM, Schneider JA, Bedell BJ, Beach TG, Bilker WB, Mintun MA, Pontecorvo MJ, Hefti F, Carpenter AP, Flitter ML, et al. Use of florbetapir-PET for imaging beta-amyloid pathology. Jama. 2011;305:275-83.

47. Hansson O, Seibyl J, Stomrud E, Zetterberg H, Trojanowski JQ, Bittner T, Lifke $V$, Corradini V, Eichenlaub U, Batrla R, et al. CSF biomarkers of Alzheimer's disease concord with amyloid- $\beta$ PET and predict clinical progression: a study of fully automated immunoassays in BioFINDER and ADNI cohorts. Alzheimers Dement. 2018;14:1470-81.

48. Landau SM, Mintun MA, Joshi AD, Koeppe RA, Petersen RC, Aisen PS, Weiner MW, Jagust WJ, Alzheimer's Disease Neuroimaging I. Amyloid deposition, hypometabolism, and longitudinal cognitive decline. Ann Neurol. 2012;72:578-86.

49. Jagust WJ, Bandy D, Chen K, Foster NL, Landau SM, Mathis CA, Price JC, Reiman EM, Skovronsky D, Koeppe RA, Alzheimer's Disease Neuroimaging I. The Alzheimer's Disease Neuroimaging Initiative positron emission tomography core. Alzheimer's \& Dementia. 2010;6:221-9.

50. Jack CR Jr, Lowe VJ, Senjem ML, Weigand SD, Kemp BJ, Shiung MM, Knopman DS, Boeve BF, Klunk WE, Mathis CA, Petersen RC. 11C PiB and structural MRI provide complementary information in imaging of Alzheimer's disease and amnestic mild cognitive impairment. Brain. 2008; 131:665-80.

51. Zhang Z, Parker RMA, Charlton CMJ, Leckie G, Browne WJ. R2MLwiN: a package to run MLwiN from within R. J Statistical Software. 2016;1(10):2016.

52. Zetterberg H, Wilson D, Andreasson U, Minthon L, Blennow K, Randall J, Hansson O. Plasma tau levels in Alzheimer's disease. Alzheimers Res Ther. 2013:5:9-9.

53. Morris JC, Roe CM, Xiong C, Fagan AM, Goate AM, Holtzman DM, Mintun MA. APOE predicts amyloid-beta but not tau Alzheimer pathology in cognitively normal aging. Ann Neurol. 2010;67:122-31.

54. Qiu C, Bäckman L, Winblad B, Agüero-Torres H, Fratiglioni L. The influence of education on clinically diagnosed dementia incidence and mortality data from the Kungsholmen Project. Arch Neurol. 2001;58:2034-9.

55. Meng X, D'Arcy C. Education and dementia in the context of the cognitive reserve hypothesis: a systematic review with meta-analyses and qualitative analyses. PLoS One. 2012;7:e38268.

56. Wilson RS, Yu L, Lamar M, Schneider JA, Boyle PA, Bennett DA: Education and cognitive reserve in old age. Neurology. 2019. https://doi.org/10.1212/ WNL.0000000000007036

57. Fagan AM, Xiong C, Jasielec MS, Bateman RJ, Goate AM, Benzinger TLS, Ghetti B, Martins RN, Masters CL, Mayeux R, et al. Longitudinal change in CSF biomarkers in autosomal-dominant Alzheimer's disease. Sci Transl Med. 2014;6:226ra230. 
58. Kennedy KM, Rodrigue KM, Devous MD Sr, Hebrank AC, Bischof GN, Park DC. Effects of beta-amyloid accumulation on neural function during encoding across the adult lifespan. Neurolmage. 2012;62:1-8.

59. Amariglio RE, Becker JA, Carmasin J, Wadsworth LP, Lorius N, Sullivan C, Maye JE, Gidicsin C, Pepin LC, Sperling RA, et al. Subjective cognitive complaints and amyloid burden in cognitively normal older individuals. Neuropsychologia. 2012;50:2880-6.

60. Farrell ME, Kennedy KM, Rodrigue KM, Wig G, Bischof GN, Rieck JR, Chen X, Festini SB, Devous MD Sr, Park DC. Association of longitudinal cognitive decline with amyloid burden in middle-aged and older adults: evidence for a dose-response relationship. JAMA Neurol. 2017;74:830-8.

61. Stomrud E, Minthon L, Zetterberg H, Blennow K, Hansson O. Longitudinal cerebrospinal fluid biomarker measurements in preclinical sporadic Alzheimer's disease: a prospective 9-year study. Alzheimers Dement (Amst). 2015;1:403-11.

62. Jicha GA, Carr SA. Conceptual evolution in Alzheimer's disease: implications for understanding the clinical phenotype of progressive neurodegenerative disease. J Alzheimer's Disease. 2010;19:253-72.

63. Dubois B, Feldman HH, Jacova C, Hampel H, Molinuevo JL, Blennow K, DeKosky ST, Gauthier S, Selkoe D, Bateman R, et al. Advancing research diagnostic criteria for Alzheimer's disease: the IWG-2 criteria. Lancet Neurol. 2014;13:614-29.

64. Amieva H, Le Goff M, Millet X, Orgogozo JM, Peres K, Barberger-Gateau P, Jacqmin-Gadda H, Dartigues JF. Prodromal Alzheimer's disease: successive emergence of the clinical symptoms. Ann Neurol. 2008:64:492-8.

65. Albert MS, Dekosky ST, Dickson D, Dubois B, Feldman HH, Fox NC, Gamst A, Holtzman DM, Jagust WJ, Petersen RC, et al. The diagnosis of mild cognitive impairment due to Alzheimer's disease: recommendations from the National Institute on Aging-Alzheimer's Association workgroups on diagnostic guidelines for Alzheimer's disease. Alzheimer's Dementia. 2011;7: 270-9.

66. Sabuncu MR, Desikan RS, Sepulcre J, Yeo BT, Liu H, Schmansky NJ, Reuter M, Weiner MW, Buckner RL, Sperling RA, Fischl B. The dynamics of cortical and hippocampal atrophy in Alzheimer disease. Arch Neurol. 2011;68:1040-8.

67. Bateman RJ, Blennow K, Doody R, Hendrix S, Lovestone S, Salloway S, Schindler R, Weiner M, Zetterberg H, Aisen P, Vellas B. Plasma biomarkers of AD emerging as essential tools for drug development: an EU/US CTAD task force report. J Prev Alzheimers Dis. 2019;6:169-73.

68. Preische O, Schultz SA, Apel A, Kuhle J, Kaeser SA, Barro C, Gräber S, KuderBuletta E, LaFougere C, Laske C, et al. Serum neurofilament dynamics predicts neurodegeneration and clinical progression in presymptomatic Alzheimer's disease. Nat Med. 2019;25:277-83.

69. Mattsson N, Andreasson U, Zetterberg H, Blennow K. Association of plasma neurofilament light with neurodegeneration in patients with Alzheimer disease. JAMA Neurol. 2017;74:557-66.

70. Lewczuk P, Ermann N, Andreasson U, Schultheis C, Podhorna J, Spitzer P, Maler JM, Kornhuber J, Blennow K, Zetterberg H. Plasma neurofilament light as a potential biomarker of neurodegeneration in Alzheimer's disease. Alzheimers Res Ther. 2018:10:71.

71. Mattsson N, Cullen NC, Andreasson U, Zetterberg H, Blennow K. Association Between Longitudinal Plasma Neurofilament Light and Neurodegeneration in Patients With Alzheimer Disease. JAMA Neurol. 2019;76(7):791-9. https:/ doi.org/10.1001/jamaneurol.2019.0765.

72. Buchhave P, Minthon L, Zetterberg H, Wallin ÅK, Blennow K, Hansson O. Cerebrospinal fluid levels of $\beta$-amyloid 1-42, but not of tau, are fully changed already 5 to 10 years before the onset of Alzheimer dementia. Arch Gen Psychiatry. 2012;69:98-106.

73. Rowe CC, Ellis KA, Rimajova M, Bourgeat P, Pike KE, Jones G, Fripp J, Tochon-Danguy H, Morandeau L, O'Keefe G, et al. Amyloid imaging results from the Australian Imaging, Biomarkers and Lifestyle (AIBL) study of aging. Neurobiol Aging. 2010;31:1275-83.

74. Insel PS, Ossenkoppele R, Gessert D, Jagust W, Landau S, Hansson O, Weiner MW, Mattsson N. Time to amyloid positivity and preclinical changes in brain metabolism, atrophy, and cognition: evidence for emerging amyloid pathology in Alzheimer's disease. Front Neurosci. 2017;11:281.

\section{Publisher's Note}

Springer Nature remains neutral with regard to jurisdictional claims in published maps and institutional affiliations.

\section{Ready to submit your research? Choose BMC and benefit from:}

- fast, convenient online submission

- thorough peer review by experienced researchers in your field

- rapid publication on acceptance

- support for research data, including large and complex data types

- gold Open Access which fosters wider collaboration and increased citations

- maximum visibility for your research: over $100 \mathrm{M}$ website views per year

At BMC, research is always in progress.

Learn more biomedcentral.com/submissions 